\title{
Hypokalemic quadriparesis due to renal tubular acidosis in a patient with Sjögren's syndrome: A case series
}

\author{
Abhishek Maskey, ${ }^{1 *}$ Neeraj Singh, ${ }^{1}$ Santosh Gautam, ${ }^{2}$ Prashant Bhattarai, ${ }^{1}$ Prakash Poudyal, ${ }^{1}$ \\ Dhiraj Narayan Manandhar, ${ }^{1}$ Pramod Kumar Chhetri ${ }^{1}$
}

${ }^{1}$ Department of Nephrology; ${ }^{2}$ Department of Internal Medicine;

Nepal Medical College and Teaching Hospital, Kathmandu, Nepal.

\section{Accepted on}

December 29th, 2013

Dol Name

http://dx.doi.org/10.3126/jaim.v3i1.10701

\section{Keywords}

Key words: Hypokalemia, quadriparesis, renal tubular acidosis type I, Sjögren's syndrome

\section{Citation}

Abhishek Maskey, Neeraj Singh, Santosh Gautam, Prashant Bhattarai, Prakash Poudyal, Dhiraj Narayan Manandhar, Pramod Kumar Chhetri. Hypokalemic quadriparesis due to renal tubular acidosis in a patient with Sjögren's syndrome: A case series. Journal of Advances in Internal Medicine 2014;03(01):30-33.

\begin{abstract}
We report two cases of female patients presented with hypokalemia secondary to renal tubular acidosis. Sjögren's syndrome was diagnosed in both the patients on the basis of histopathological and autoantibodies tests. The patients were treated with potassium and bicarbonate supplementation. Renal involvement in Sjögren's syndrome is not uncommon and may precede sicca complaints. The pathology in most cases is a tubulointerstitial nephritis causing distal renal tubular acidosis and rarely, hypokalemic paralysis. The complications of renal tubular acidosis include life threatening hypokalemia, nephrolithiasis, chronic renal failure, growth retardation and osteomalacia. These consequences can be avoided if the diagnosis is made early and lifelong potassium and alkali supplementation is initiated. Primary Sjögren's syndrome should be considered in women with acute weakness and hypokalemia.
\end{abstract}

\section{INTRODUCTION}

Sjögren's syndrome is a slowly progressing autoimmune disease characterized by lymphocytic infiltration of the exocrine glands, mainly the lacrimal and salivary glands, resulting in their impaired secretory function. Systemic involvement and symptoms of cutaneous, respiratory, renal, hepatic, neurologic, and vascular systems often occur. ${ }^{1}$ Renal involvement is a well recognized extra glandular manifestation and may be related to chronic interstitial nephritis. The resultant tubular dysfunction may manifest as proximal or distal renal tubular acidosis, tubular proteinuria or nephrogenic diabetes insipidus. ${ }^{2,3}$ Renal tubular acidosis occurs almost $25-40 \%$ of patients with Sjogren syndrome. ${ }^{4}$ renal manifestations like hypokalemic periodic paralysis, urolithiasis, or osteomalacia are uncommon. ${ }^{1}$ Hypokalemic paralysis secondary to Sjogren's syndrome with distal renal tubular acidosis is rarely seen in our patients population. We report a case of flaccid quadriparesis in a patient with Sjogren syndrome. Our aim is to emphasize one of the rare presentations of Sjogren's syndrome but also to highlight the favourable response to potassium and bicarbonate therapy.

\section{Clinical history and initial laboratory data:}

\section{Case 1:}

A 28 years female was presented to Nepal Medical College and Teaching Hospital on 22 nd April 2013 (2070/01/09) with history of weakness of all four limbs associated with dull aching pain in thighs and calves for 2 days. On the day of admission the patient also complained of difficulty in breathing and speaking long sentences. There was no history of difficulty in swallowing, no history of diplopia, seizures, blurring of vision, facial asymmetry, sensory symptoms, bladder or bowel complaints. She did not give any history of

\footnotetext{
* Corresponding author

Dr. Abhishek Maskey

DM resident, Department of Nephrology

Nepal Medical College Teaching Hospital, Jorpati, Kathmandu

Email: maskey21@hotmail.com.
} 
recent drug intake as well as vaccination. She reported similar but less severe illness 4 months previously. This episode was treated with intravenous fluid at local hospital and weakness resolved completely. A more focused interview uncovered 3-4 years of keratoconjunctivitis, but no history of skin rash, photosensitivity and arthritis.

On examination, patient was conscious, cooperative and oriented. Vital signs showed blood pressure recorded was 110/80 mm Hg; heart rate 104 bpm, regular; respiratory rate $30 / \mathrm{min}$ and body temperature was normal. On central nervous system examination, higher mental function, cranial nerves were normal. Lower limbs had mild tenderness. Tone was decreased in all four limbs. Power was reduced in all four limbs to $3 / 5$. Plantar reflexes were flexor bilaterally. Tendon reflexes except for ankle and knee reflexes were diminished. No abnormalities were detected on examination of the heart, lungs and abdomen.

Based on lab data (Table 1), we considered that flaccid quadriparesis was induced by severe hypokalemia. The differential diagnosis for the hypokalemia included renal tubular acidosis and hypokalemic periodic paralysis. The latter was excluded once all the data were available. Shortly after admission, the patient was transferred to Intensive care unit where an infusion of potassium was started. This was continued until her serum potassium was normalized and her weakness improved. Urinary potassium was high despite severe hypokalemia. She was then transferred to our department to investigate the cause of renal tubular acidosis.

Additional laboratory tests are summarized in Table 2. Ultrasonograph of abdomen showed normal sized kidney without any evidence of calcification or any obstruction. Schirmer test revealed moderate to severe dry eyes indicating insufficient production of tears. Labial gland biopsy revealed fibrous tissue along with scattered lymphocytes suggestive of Sjögren's syndrome. After diagnosis was confirmed, the patient was started on oral sodium bicarbonate therapy and artificial tears for the dry eyes.

Case 2:

Another case was referred to us for the evaluation of hypokalemia. She was a $65 y$ r old female. She had presented with generalized weakness, low backache and she was on potassium supplementation for less than a year duration. She was kept on aldactone as well to maintain the serum potassium level. But once the supplements were withdrawn, the potassium level would fall down. When she presented to us she didn?t have objective weakness of the lower limbs but she did complain of weakness of the lower limbs in the past, however documentation of degree of weakness was not mentioned in the notes. Previous evaluation revealed that she had osteopenia. Cranial nerves examination was normal. Her bowel and bladder habit was normal. She didn?t have sensory symptoms. She didn?t give history of rash, arthritis but had seborrhoeic keratitis with positive Schirmer test, and solar maculopathy. She was managed initially with potassium supplementation and detailed evaluation was done. The reports of blood tests are tabulated in Table $1 \& 2$. The tests and gingival biopsy underwent thereafter revealed condition suggestive of Sjögren's syndrome. She is now on sodium bicarbonate and minimal potassium supplementation and artificial tear for the dry eyes and sun protective glasses for solar maculopathy. [Table:1)

\begin{tabular}{lll}
\hline \multicolumn{1}{c}{ Parameters } & \multicolumn{1}{c}{ Case 1 } & \multicolumn{1}{c}{ Case 2 } \\
\hline S. Sodium & $140 \mathrm{meq} / \mathrm{l}$ & 141 \\
S. Potassium & $1.9 \mathrm{meq} / \mathrm{l}$ & 2.8 \\
Blood urea & $18 \mathrm{mg} / \mathrm{dl}$ & 32 \\
S. Creatinine & $0.8 \mathrm{mg} / \mathrm{dl}$ & 1.4 \\
Hemoglobin & $11.4 \mathrm{gm} \%$ & 11.0 \\
Total Leukocyte count & $4500 / \mathrm{cc}$ & 4600 \\
Platelets count & $390000 / \mathrm{cc}$ & 166000 \\
S. Calcium & $7.1 \mathrm{mg} / \mathrm{dl}$ & 9.7 \\
S. Phosphorus & $1.5 \mathrm{mg} / \mathrm{dl}$ & 3.3 \\
Arterial blood gas analysis & & \\
$\mathrm{pH}$ & 7.34 & 7.32 \\
$\mathrm{pCO}_{2}$ & $26.8 \mathrm{~mm} \mathrm{Hg}$ & 25 \\
$\mathrm{pO}_{2}$ & $116.1 \mathrm{~mm} \mathrm{Hg}$ & 71 \\
$\mathrm{HCO}_{3}$ & $14.1 \mathrm{mEq} / \mathrm{l}$ & 13 \\
Urinary Potassium & $520 \mathrm{meq} / \mathrm{l}$ & 23.85 \\
\hline
\end{tabular}

[Table:2)

\begin{tabular}{|lcc|}
\hline Tests & Case 1 & Case 2 \\
\hline Anti nuclear antibody & $\begin{array}{c}\text { Positive to } \\
\text { a titre of } \\
1 / 640 \text { with } \\
\text { a speckled } \\
\text { pattern }\end{array}$ & $\begin{array}{c}\text { Positive to } \\
\text { a titre of } \\
1 / 320 \text { with } \\
\text { a speckled } \\
\text { pattern }\end{array}$ \\
Anti double stranded DNA & Negative & $\begin{array}{c}\text { Equivocal } \\
\text { (30 IU/ml) }\end{array}$ \\
Anti Ro/SS-A antibody & Positive & Positive \\
Anti- La/SS-B antibody & Positive & Positive \\
Rheumatoid factor & Negative & Negative \\
Hepatitis B, C, HIV I \&II and & Negative & Negative \\
VDRL serology & & \\
Thyroid function test & Normal & Normal \\
USG KUB & Normal & Normal \\
\hline
\end{tabular}

Discussion: Sjögren's syndrome is systemic chronic inflammatory disorder characterized by lymphocytic infiltration in exocrine gland. There are two age peaks of primary Sjögren's syndrome, with the first after menarche during the $20 \mathrm{~s}$ to $30 \mathrm{~s}$ and the second after menopause in the mid-50s. ${ }^{1}$ The syndrome can present either alone as primary Sjogren's syndrome or as secondary Sjögren's syndrome in the context of underlying connective tissue diseases such as rheumatic arthritis, 
systemic lupus erythematosus. ${ }^{5}$ Primary Sjögren's syndrome is a systemic autoimmune disorder with a population prevalence of about $0.5 \%$ and female preponderance (female: male ratio of 9: 1). ${ }^{6}$ Extraglandular manifestation of Sjögren's syndrome affects kidney, liver, lungs, pancreas and nervous system.

Renal involvement is not uncommon and may precede sicca complaints. The pathology in most cases is tubulointerstital nephritis causing among other distal renal tubular acidosis and

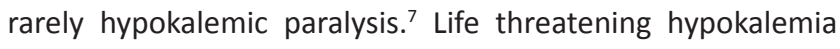
with muscle paralysis is most serious clinical consequence of distal renal tubular acidosis. ${ }^{8}$

The hallmark of renal tubular acidosis (RTA) is a low rate of ammonium excretion by the kidneys in the presence of chronic metabolic acidosis. We did not have detailed urine chemistry available and could not accurately determine urine ammonium concentration but it is safe to assume that ammonium excretion is low if the urine has an alkaline $\mathrm{pH}$. When the urine $\mathrm{pH}$ is high, the mechanism which might cause distal RTA include reduced activity of $\mathrm{H}^{+}$- ATPase pumps in the distal nephrons, back leak of secreted $\mathrm{H}^{+}$as seen with amphotericin $\mathrm{B}$ treatment and high secretion of $\mathrm{HCO}_{3}$ as seen in some patients with Southeast Asian ovalocytosis. The high urine $\mathrm{pH}$ and/or bicarbonaturia cause renal potassium wasting and leads to hypokalemia. ${ }^{9}$ Another complication of the high urinary $\mathrm{pH}$ is the increased risk of renal $\mathrm{CaHPO} 4$ deposition with nephrocalcinosis and progressive renal insufficiency.

Although distal RTA is very common in Sjögren's syndrome it is usually asymptomatic and goes undetected in many cases. ${ }^{10}$ Our patients also showed metabolic acidosis with alkaline urine and severe hypokalemia. ${ }^{11}$ The pathogenesis of distal RTA in Sjögren's syndrome is not very clear. It appears to be the result of an immunologic assault on the collecting tubules, causing failure to insert $\mathrm{H}^{+}$- ATPase into the apical membrane of intercalated cells. ${ }^{12,13}$ Another study found high levels of anti carbonic anhydrase antibodies affecting the function of carbonic anhydrase in cortical collecting duct. ${ }^{14} \mathrm{~A}$ further possibility is that the $\mathrm{Cl}^{-} / \mathrm{HCO}_{3}{ }^{-}$exchanger is misdirected to the apical membrane as in Southeast Asian ovalocytosis,

\section{REFERENCES}

1. Fox IR. Sjogren's syndrome. Lancet 2005;366:321-31. http://dx.doi.org/10.1016/S0140-6736(05)66990-5

2. Bossini N, Savoldi S, Franceschini F, et al. Clinical and morphological features of kidney involvement in primary Sjogren's syndrome. Nephrol Dial Transplant 2001;16:2328-36.

http://dx.doi.org/10.1093/ndt/16.12.2328

3. Hiroshi O, Imai H, Kodama T, et al. Severe hypolkalaemia and respiratory arrest due to renal tubular acidosis in a patient with Sjogrens syndrome. Nephrol Dial transplant 1999;14:2201-3. causing bicarbonate secretion in the distal tubule. ${ }^{9}$

On presentation, our $1^{\text {st }}$ patient was initially thought to have hypokalemic periodic paralysis as she described asimilar episodes of weakness in the past. Her thyroid function was normal, excluding the thyrotoxic form of periodic paralysis. The history of dry eyes, positive autoantibodies tests and the labial biopsy allowed us to make the diagnosis of Sjögren's syndrome while the metabolic acidosis, high urine $\mathrm{pH}$ and renal potassium wasting led to the diagnosis of distal RTA in both the cases.

Hypokalemic paralysis in association with Sjögren's syndrome is rare and only occasionally is the manifestation that brings a patient to a doctor. ${ }^{8} \mathrm{~A}$ renal biopsy is unnecessary in the patients with suspected tubulointerstitial nephritis. ${ }^{15}$ We did not perform kidney biopsy in these patient as there were no features suggestive of glomerular involvement such as heavy proteinuria or loss of renal function. Most kidney biopsies done in patients with Sjögren's syndrome have revealed lymphocytic and plasma cell infiltration of interstitium. ${ }^{16}$

Correction of hypokalemia and alkali replacement are standard therapy in all patients and there is no need to use immunosuppressive therapy for the RTA. Hypokalemia should be corrected first because alkaline replacement can aggravate hypokalemia by increasing renal potassium losses. Combination of corticosteroids and other immunosuppressants have been used when the renal biopsy has confirmed significant glomerular disease. ${ }^{17}$

\section{CONCLUSION}

Hypokalemia should always be considered in patients presenting with muscular weakness. When high urinary potassium losses are present, renal tubular acidosis must be included in differential diagnosis. If RTA is confirmed, Sjögren's syndrome should be considered as an underlying cause. Timely diagnosis and appropriate treatment of patients with this form of distal RTA can prevent morbidity from hypokalemia and nephrocalcinosis.

\section{http://dx.doi.org/10.1093/ndt/14.9.2201}

4. Goules A, Masouridi S, Tzioufas AG, et al. Clinically significant and biopsy-documented renal involvement in primary Sjogren's syndrome. Medicine (Baltimore) 2000;79:241-9.

http://dx.doi.org/10.1097/00005792-20000700000005

5. Khandelwal D, Bhattachary S, Khadgawat R, et al. Hypokalemic paralysis as a presenting manifestation of primary Sjögren's syndrome: A report of two cases. Indian J Endocrinol Metab 2012;16:853-85.

http://dx.doi.org/10.4103/2230-8210.100684 
6. Bowman SJ, Ibrahim GH, Hodams G, et al. Estimating the prevalence among Caucasian women of primary Sjogren syndrome in two general practice in Birmingham UK. Scand J Rheumatol 2004;33:39-43.

http://dx.doi.org/10.1080/03009740310004676

7. Hakkı Y, Mustafa K, Mustafa O, et al. Hypokalemic periodic paralysis in Sjogren's syndrome secondary to distal renal tubular acidosis. Rheumatol Int 2013;33:1879-82.

http://dx.doi.org/10.1007/s00296-011-2322-z

8. Palkar AV, Pillai S, Rajadhyaksha. Hypokalemic quadriparesis in Sjogren syndrome. Indian J Nephrol 2011;21;190-2.

9. Halperin ML, Goldstein MB, Kamel KS. Fluid, electrolyte and acid base physiology: A problem- based approach. Ed 4th, Philadelphia PA, Saunders/ Elsevier, 2010.

10. Takemoto F, Hoshino J, Sawa N, et al. Autoantibodies against carbonic anhydrase $\mathrm{II}$ are increased in renal tubular acidosis associated with Sjogren syndrome. Am J Med 2005;118:181-4.

http://dx.doi.org/10.1016/j.amjmed.2004.07.049

11. Gaurang V, Swapnil G. Sjogren's syndrome with distal renal tubular acidosispresenting as hypokalaemic paralysis. BMJ Case Reports 2012;10:1136.
12. Thomas D, Dubose Jr. A 42 year old woman with Flaccid Paralysis. Acid base and electrolyte teaching case. Am J Kidney disease 2009;54:965-9.

http://dx.doi.org/10.1053/j.ajkd.2009.07.006

13. Joo KW, Jeon US, Han JS, et al. Absence of H(+)-ATPase in the intercalated cells of renal tissues in classic distal renal tubular acidosis. Clin Nephrol 1998;49:226-31.

14. Soy M, Pamuk ON, Gerenli M, et al. A primary Sjögren's syndrome patient with distal renal tubular acidosis, who presented with symptoms of hypokalemic periodic paralysis: report of a case study and review of the literature. Rheumatol Int 2005;26:86-9.

http://dx.doi.org/10.1007/s00296-005-0587-9

15. Ramos CA, Tzioufas GA, Font J. Primary Sjogren's syndrome: New clinical and therapeutic concepts. Ann Rheum Dis 2005;64:347-54.

http://dx.doi.org/10.1136/ard.2004.025676

16. Reddy KS, Jha V, Nada R, et al. Respiratory paralysis in Sjogren syndrome with normal renal function. Natl Med J India 2003;16:253-4

17. Maripuri S, Grande JP, Osborn TG. Renal involvement in primary Sjogren's syndrome: a clinicopathologic study. Clin J Am Soc Nephrol 2009;4:1423-31. http://dx.doi.org/10.2215/CJN.00980209 